\title{
Simulation of screw dislocations in wadsleyite
}

\author{
Andrew M. Walker \\ Department of Earth Sciences, University College London, Gower Street, London, \\ WC1E 6BT, UK
}

Received: date / Revised version: date

\begin{abstract}
The structure and energies of the cores of [100] and [001] screw dislocations in wadsleyite $\left(\beta-\mathrm{Mg}_{2} \mathrm{SiO}_{4}\right)$ are calculated using a cluster-based combined elastic-atomistic method and a new parameterized interatomic potential model. For a core radius of $10 \AA$, core energies are found to be 2.5 and $4.4 \mathrm{eV} / \AA$ for the [100] and [001] dislocations, respectively. Both dislocations are associated with significant non-elastic displacement fields extending beyond the core with a radial component towards the dislocation line. The core of the [100] dislocation contains tetrahedrally coordinated magnesium, has a simple 2D structure and is spread parallel to (011) in a manner that suggests high mobility. In contrast, the core of the [001] dislocation has an extended and complex $3 \mathrm{D}$ structure involving the formation a large $\mathrm{Si}_{6} \mathrm{O}_{19}$ unit twisted around the dislocation line. This implies that
\end{abstract}

Correspondence to: Andrew M. Walker, e-mail: a.walker@ucl.ac.uk, phone: +44 (0)20 7679 2425, fax: +44(0)20 76792433 
movement of the [001] dislocation will be inhibited by the need to cleave Si-O bonds. These observations, combined with the anomalously low core energy of the [100] dislocation, explain the regular occurrence of [100] dislocations and very rare observation of [001] dislocations in experimentally deformed wadsleyite samples.

Key words wadsleyite, screw dislocations, deformation, atomic scale modeling, dislocation energy

\section{Introduction}

The motion of dislocations in mantle minerals plays a key role in determining their rheology and, in turn, controlling the vigor and style of the solid state convection that drives plate tectonics and is responsible for many of the geological processes expressed at the Earth's surface. These one-dimensional extended defects can also contribute to seismic dispersion and attenuation (Jackson, 2007; Karato and Spetzler, 1990) and provide pathways for rapid diffusion of trace and major elements into and out of the interior of minerals. Many of the properties of dislocations can be understood in terms of imperfections in an elastic continuum but others, such as their mobility and effect on diffusivity, are determined entirely by the detailed atomic scale structure of the dislocation core. Although experimental methods are approaching this resolution in some cases (Johnson et al., 2004; Jia et al., 2005), only computer simulation at the atomic scale can fully resolve the 
structure of dislocation cores needed to understand these processes. Most modeling of dislocation cores in minerals has been performed by parameterizing the Peierls-Nabarro model (Peierls, 1940; Nabarro, 1947) using the energies of generalized stacking faults from density functional theory to introduce nonlinear forces at the atomic scale into a continuum model of the dislocation. This approach, which has the key advantage of a predictive and ab-initio description of the atomic interactions, introduces, among others, the significant constraint of a planar dislocation core (e.g. Schoeck, 2005). Nevertheless, the Peierls-Nabarro approach has been applied to many minerals including $\mathrm{MgSiO}_{3}$ perovskite, post-perovskite and analogue materials (Carrez et al., 2007a,b; Ferré et al., 2007, 2008, 2009a,b), $\alpha-\mathrm{Mg}_{2} \mathrm{SiO}_{4}$ forsterite (Durinck et al., 2005a,b, 2007) and $\gamma-\mathrm{Mg}_{2} \mathrm{SiO}_{4}$ ringwoodite (Carrez et al., 2006). Alternatives to the Peierls-Nabarro model involve explicit calculation of the dislocation core structure using atomic scale simulation. These models can make use of either one-dimensional boundary conditions to simulate an isolated dislocation (the 'cluster' approach used in this work) or three-dimesnional boundary conditions to simulate an infinite array of dislocations (the 'dipole' approach). In the latter case it is important to consider interactions between the dislocation core fields (Clouet et al., 2009) and to include the contribution from core traction in the dislocation formation energy (Clouet, 2009).

Wadsleyite, $\beta-\mathrm{Mg}_{2} \mathrm{SiO}_{4}$, is a major component of the upper part of the mantle's transition zone. It is stable at pressures intermediate to the low 
pressure olivine structure and the high pressure ringwoodite (spinel) structure and accounts for around $40 \%$ of the mantle by volume for a pyrolitic composition at depths between 410 and $520 \mathrm{~km}$. An understanding of the deformation mechanisms of wadsleyite is particularly useful as a signature of mantle dynamics and, in particular, to discriminate between whole-mantle and layered convection. Layered convection should result in horizontal flow in the transition zone whereas whole mantle convection should lead to vertical flow in this region. If dislocation glide is the active deformation mechanism the flow pattern may be recorded as a crystallographic preferred orientation in wadsleyite. This would in turn lead to potentially observable seismic anisotropy. Tommasi et al. (2004) have shown that the weak seismic anisotropy of the transition zone (e.g. Montagner and Kennett, 1996; Trampert and van Heijst, 2002) can be explained by a crystallographic preferred orientation created by dislocation creep of wadsleyite and argue that this suggests that flow in the transition zone has a dominant horizontal component.

Wadsleyite is a spinelloid with a body-centered orthorhombic unit cell (space group Imma). The crystal structure can be understood in terms of a distorted cubic close packed array of oxygen atoms with magnesium and silicon ions embedded in tetrahedral and octahedral interstices. Alternatively, it can be viewed as consisting of $\mathrm{SiO}_{4}$ and $\mathrm{MgO}_{6}$ polyhedral units with pairs of silicate tetrahedra sharing a single vertex to form isolated $\mathrm{Si}_{2} \mathrm{O}_{7}$ groups. Studies of samples from meteorites (e.g. Price et al., 1982; 
Price, 1983; Madon and Poirier, 1983) and experiments (e.g. Kerschhofer et al., 1996, 1998) show that transformation between olivine and wadsleyite can occur via incoherent nucleation on olivine grain boundaries or by an intracrystalline mechanism involving the migration of partial dislocations. The second mechanism can result in an increase in the dislocation density during transformation from olivine to wadsleyite.

Several studies have been performed in order to determine the strength and active slip systems of wadsleyite at high temperature and pressure (Sharp et al., 1994; Dupas et al., 1994; Dupas-Bruzek et al., 1998; Chen et al., 1998; Mossenfelder et al., 2000; Nishihara et al., 2008). These have mostly been performed in multianvil apparatus adapted to impose deviatoric stress and with a starting material containing olivine. Dislocations present in recovered samples have been characterized using the transmission electron microscope. Extended defects identified by Dupas et al. (1994) and Dupas-Bruzek et al. (1998) include (010) stacking faults interpreted as growth defects, and dislocations belonging to the active [100]\{021\} and $\frac{1}{2}\langle 111\rangle\{101\}$ slip systems. At higher temperature, Sharp et al. (1994) found evidence for movement of [100] dislocations on the (010) plane. Measurements of the strength of wadsleyite shows that it is stronger than olivine (Mossenfelder et al., 2000; Nishihara et al., 2008) and that the addition of hydrogen leads to significant hydrolytic weakening (Chen et al., 1998). One difficulty with these experiments is the need to untangle microstructures formed during the deformation and transformation processes. 
Perhaps the most complete study of deformation induced microstructures in wadsleyite was undertaken by Thurel and co-workers. This series of experiments avoided problems with the transformation microstructure by first pre-transforming synthetic forsterite powder into wadsleyite. The samples were then recovered before being deformed under anisotropic compression at $1800-2000{ }^{\circ} \mathrm{C}$ and $15-19 \mathrm{GPa}$ (Thurel and Cordier, 2003) and shear at $1300{ }^{\circ} \mathrm{C}$ and $14 \mathrm{GPa}$ (Thurel et al., 2003a) in multianvil apparatus in separate experimental runs. The deformed specimens were then examined in the electron microscope. Reported slip systems from the compression experiments were [100] dislocations on the (010), (001), \{011\} and $\{021\}$ planes, $\frac{1}{2}\langle 111\rangle$ dislocations on $\{101\},[010]$ on $(001)$ and $\{101\}$, and $\langle 101\rangle$ on (010). Dislocations with a [001] Burgers vector were observed once and interpreted as being immobile. Fewer slip systems were observed following the shear experiments where only [100] and $\frac{1}{2}\langle 111\rangle$ dislocations were interpreted as being activated. Thurel et al. (2003b) explained these observations by calculating the elastic energy of the various dislocations and considering various reactions leading to the formation of partial dislocation. This showed the importance of the dissociation of dislocations to lower the elastic energy for several of the slip systems. [100] screw dislocations were particularly common and dissociation of these dislocations was not required on energetic grounds. Indeed, dissociated [100] screw dislocations were only observed very occasionally. The elastic energy does not explain this observation, and the existence of a favorable core structure for the screw dislocation 
was suggested (Thurel et al., 2003b). Few [001] dislocations were observed, which suggests they have low mobility and thus do not multiply during deformation. The reason for this low mobility was unclear, but may be due an inability for it to dissociate into partials (Thurel et al., 2003b). Any study based on elastic theory alone is clearly limited by a lack of resolution of the core structure, a fact acknowledged by Thurel et al. (2003b) who conclude that it "seems necessary, however, to carry out further detailed investigation on the precise core structure of the dislocations in wadsleyite to better understand and model the plastic behaviour of this mineral". Atomic scale simulation is currently the only method that can provide the detailed and three-dimensional view of the core of dislocations needed to address this problem. The results presented below show how this detailed view can be obtained for the two non-dissociated screw dislocations and suggests why they have such different behavior.

\section{Methodology}

Models of the [100] and [001] screw dislocations in wadsleyite were constructed following the methodology described in Walker et al. (2005a). The approach combines an atomic scale model of the core with a description of the extended crystal based on continuum linear elasticity and has previously been used for models of screw dislocations in $\mathrm{MgO}$, zeolite $\mathrm{A}$, forsterite, and orthorhombic paracetamol-II (Walker et al., 2004, 2005b; Carrez et al., 
2008). The calculations were performed using parameterized interatomic potentials making use of the GULP code (Gale, 1997; Gale and Rohl, 2003).

Interactions between atoms are described using a new interatomic potential model designed to be transferable, free of discontinuities on the potential energy surface, and scalable to very large systems. To this end the model follows the general approach taken by Sanders et al. (1984) and Lewis and Catlow (1985) to produce their successful and transferable potential model, sometimes called 'THB1' in the literature. In common with this earlier model, interactions between atoms consist of a Coulomb term with atoms given formal charges, short-range Buckingham type interactions, a shell model to account for oxygen polarizability and a three-body term to account for repulsion between $\mathrm{Si}-\mathrm{O}$ covalent bonds in silicate tetrahedra. The key difference in the formulation of the new model is that the Coulomb term is evaluated using a spherically truncated, pairwise, real space summation (Wolf et al., 1999). This allows calculations using the model to make use of physically intuitive and easily parallelizable spatial locality to realize linear scaling with system size (e.g. Fennell and Gezelter, 2006). Additionally, smoothness of the potential is imposed by including tapering of the pairwise short range terms using a cosine function and by replacing the harmonic three-body term, which has a radial discontinuity, with the exponentialharmonic form (Gale and Rohl, 2003), which smoothly decays as the $\mathrm{Si}-\mathrm{O}$ separation increases. 
A three stage approach is taken to constructing the models of the dislocations. First a charge neutral disk-shaped simulation cell containing the defect-free crystal structure is constructed with a one-dimensional periodic repeat direction, labeled $z$, parallel to the dislocation line (and, for a screw dislocation, the Burgers vector). The [010] direction is always orientated along the $y$ axis of the simulation cell with the $x$ axis parallel to the third crystallographic direction. The simulation cell radius is chosen as $80 \AA$ to accommodate relaxation around the core and the height is equal to the length of the Burgers vector. Including the oxygen cores and shells, the simulation cells for the [100] and [001] dislocations contain 19259 and 28300 particles, respectively.

Charge neutrality of the simulation cells is imposed by building the simulation cell out of charge neutral polyhedral units centered on cations. Vertex oxygen atoms are assigned a fractional negative charge to balance the positive charge on the cation. Overlapping oxygen atoms are merged and the charges summed so that the total charge on any oxygen within the simulation cell is -2 . Charges on the oxygen atoms around the fringe of the cell are not, therefore, assigned the full charge and the total charge of the cell is zero. For forsterite (Walker et al., 2005b), assigning fractional charges is straight-forward as each oxygen belongs to one silica tetrahedra (contributing a charge of -1) and three magnesium octahedra (each contributing a charge of $\left.-\frac{1}{3}\right)$. This gives the correct formal charge on the oxygen and neutral polyhedra. For wadsleyite one oxygen atom, $\mathrm{O}(1)$, belongs to five $\mathrm{Mg}$ 
octahedra and no Si tetrahedra while the $\mathrm{Si}-\mathrm{O}-\mathrm{Si}$ bridging oxygen, $\mathrm{O}(2)$, belongs to two tetrahedra and one octahedra. In order to ensure charge neutrality for this structure the $\mathrm{O}(2)$ atoms are artificially assumed not to contribute to the octahedra while the $\mathrm{O}(1)$ atoms are assigned extra negative charge (total contribution of $-\frac{2}{3}$ ). This ensures that all polyhedra are charge neutral and all oxygen atoms in the interior of the simulation cell have the correct formal charge.

The second stage involves introducing the dislocation based on anisotropic linear elasticity (Steeds, 1973). Each model atom in the simulation cell is moved as if it was embedded in an infinite elastic medium which is deformed to introduce the dislocation. For the two cases considered in this work the elastic displacement, $u_{z}$, at a point $(x, y)$ is entirely parallel to the Burgers vector, $b$, and is given by:

$$
u_{z}=\frac{b}{2 \pi} \arctan \left(\sqrt{\frac{S_{44}}{S_{55}}} \frac{y}{x}\right)
$$

where $S_{44}$ and $S_{55}$ are components of the reduced elastic compliance matrix which is in turn calculated from the full elastic compliance matrix $\left(s_{l m}\right)$ :

$$
S_{l m}=s_{l m}-\frac{s_{3 l} s_{3 m}}{s_{33}}
$$

with the $x_{3}$ axis orientated along the Burgers vector and the $x_{1}$ and $x_{2}$ axes orientated along the two perpendicular crystallographic axes. Because the elastic displacement field for a screw dislocation has a discontinuity of $\frac{1}{2} b$ across the origin it is important to select a location for the origin such that bonds are not broken at this stage of the calculation. This was achieved by 
locating the origin along $\left(0, \frac{1}{8}\right)$ for the [100] dislocation (Figure 1$)$ and $\left(\frac{1}{4}\right.$, 0) for the [001] dislocation (Figure 2). After introducing the dislocation all $\mathrm{Si}-\mathrm{O}$ bonds are preserved.

The final step needed to generate a model of the dislocation core is to allow the atoms to move to find a low energy configuration. This process corrects for non-linear elasticity close to the core, allows the possibility of inhomogeneous strain, accounts for the atomic scale structure of the core and allows the reconstruction of the core to cause deformation of the surrounding crystal (Walker et al., 2005a). The energy was minimized using a limited memory BFGS optimizer (Nocedal, 1980) with the leading 50 diagonals of the hessian matrix included in the calculation. A $30 \AA$ thick outer rim of atoms were held fixed in the configuration predicted by linear elasticity to simulate the presence of an extended crystal. Atoms within 50 $\AA$ of the center of the cell were allowed to move to energy minimum. The fact that the outer rim of atoms is thicker than the real space Coulomb cutoff radius means that the mobile atoms do not interact with the edge of the atomistic model.

Once an energy minimum has been located various techniques described below are used to explore the structure and properties of the core. The optimized structure can also be used to extract the formation energy of the dislocation. It is first worth noting that, unlike in the case of point defects (where there is a unique defect formation energy) or surfaces (where there is a surface energy per unit area), there is not a unique dislocation energy per 
unit length of dislocation. The formation energy includes an elastic contribution arising from elastic strain distributed across the extended crystal and is thus a function of crystal size expressed as distance from the dislocation line. The total dislocation formation energy, $E$, stored within a cylindrical crystal of radius $r$ is given by:

$$
E(r)=E_{\text {core }}+\frac{K b^{2}}{4 \pi} \ln \left(\frac{r}{r_{0}}\right)
$$

where $r_{0}$ is the size of the dislocation core with energy $E_{\text {core }}$ and $b$ is the length of the Burgers vector. $K$ is an energy factor equal to the shear modulus in the isotropic case and given by:

$$
K=\left(S_{44} S_{55}\right)^{-\frac{1}{2}}
$$

for the symmetry of the [100] and [001] dislocations in wadsleyite. In order to calculate $E(r)$ for a particular radius the model derived from the energy minimization procedure is divided into two parts. Region I contains all atoms found closer to the origin than $r$ while the outer atoms are assigned to region II. The energy of the simulation cell containing the dislocation is then partitioned into interactions between atoms within region I, Ed(11), interactions between atoms in region 2, $E d(22)$ and interactions between the two regions, $E d(12)$ and $E d(21)$. Equivalent energies for the perfect system partitioned in the same manner, $E p(11), E p(22), E p(12)$ and $E p(21)$, are calculated. It is essential to ensure that the same distribution of atoms between the two regions is made and an atom at the edge of region $\mathrm{I}$ in the dislocated cell is also in region I for the perfect cell (so, for the perfect sys- 
tem, the cutoff radius is not the same as the radius used for the dislocated system; indeed, the boundary is not generally circular for the perfect system). The dislocation formation energy stored within region I is then given by $E(r)=(E d(11)+E d(12))-(E p(11)+E p(12)) . E(r)$ was calculated for a number of different radii and this data used to fit Equation 3 and extract a value for the energy of the dislocation core.

\section{Results}

Table 1 shows the parameters of the inter-atomic potential model relax fitted (Gale, 1996) to the structure and elastic constants of $\mathrm{MgO}$ and forsterite along with the static and high frequency dielectric constants of $\mathrm{MgO}$. As shown in Table 2, the new potential model reproduces both fitted and unfitted properties of the phases used for fitting as well as those of wadsleyite with similar fidelity to the 'THB1' model, which has been widely used for studies of point and extended defects in these minerals (e.g. Purton et al., 1997; de Leeuw et al., 2000; Walker et al., 2003, 2006, 2009). Compared to that model the new parameters generally lead to slightly softer elastic constants and larger cell volumes, but these differences are small when compared to the deviation between the calculated values and experimental data. For $\mathrm{MgO}$ both models are limited to simple pairwise interactions and are therefore not capable of reproducing the Cauchy violation. Neither model was fitted to the structure or properties of wadsleyite so the ability to reproduce the structure and elastic properties is reassuring. The new model used 
in the current work has the key advantage of allowing much more efficient calculations for the large simulation cells needed for models of extended defects.

The key information that can be extracted from the calculations is the detailed, atomic scale, structure of each dislocation's core out to a radius of $50 \AA$. This can be viewed in a several ways and on different length-scales. On the shortest scale an analysis of the bonding and atomic structure close to the core is of interest. For the [100] screw dislocation (Figure 3) this shows changes to the $\mathrm{Si}_{2} \mathrm{O}_{7}$ groups are minor: $\mathrm{Si}-\mathrm{O}$ bond lengths in the core change by less than $0.02 \AA$ compared to the calculated perfect structure. The $\mathrm{Si}^{-}$ $\mathrm{O}-\mathrm{Si}$ angles close to the core decreases from $124^{\circ}$ to between $123^{\circ}$ and $118^{\circ}$ and two bridging $\mathrm{Si}-\mathrm{O}(2)$ bonds in the group become slightly asymmetric with one longer and one shorter bond compared to the single bond length found in the perfect structure. The structure of the $\mathrm{MgO}_{6}$ octahedra are changed in a more noticeable manner. Five of the magnesium atoms close to the core are tetrahedrally rather than octahedrally coordinated with bond lengths of 1.8 - $2.0 \AA$. These magnesium atoms are aligned on an (011) plane passing through the core and some are further from the center of the core region than octahedrally coordinated magnesium with bond lengths similar to those found in the perfect crystal. For the [001] screw dislocation (Figure 4) there are much more dramatic changes to the structure of the core. The $\mathrm{Si}_{2} \mathrm{O}_{7}$ groups are not all preserved unaltered, indeed two $\mathrm{Si}-\mathrm{O}-\mathrm{Si}$ bonds are broken and three new ones form to create two isolated $\mathrm{SiO}_{4}$ tetrahedra and 
one large $\mathrm{Si}_{6} \mathrm{O}_{19}$ unit that is wrapped around the center of the dislocation core. Even in this deformed structure the $\mathrm{Si}-\mathrm{O}$ bond lengths are similar to those found in the perfect crystal structure apart from one abnormally long bond $(\sim 1.8 \AA)$.

A powerful way to examine the structure of the core of screw dislocations on a slightly longer length-scale is by plotting the differential displacements of adjacent atoms along the dislocation line. In this representation, rather than considering the structure of the core, displacements of atoms from their position in the bulk crystal to their position in the core are considered. Arrows are plotted between adjacent atoms with a length proportional to the difference in the component of the displacement parallel to the dislocation line. This representation clearly shows when a screw dislocation spreads onto one or more planes and allows the visualization of processes such as the spreading of $\frac{1}{2}\langle 111\rangle$ screw dislocations into the three equivalent $\{110\}$ planes in body centered cubic metals (Vitek et al., 1970). In the current case the differential displacements between oxygen atoms are plotted superimposed on the atomic scale structure in Figures 5 and 6 . For the two dislocations considered the differential displacement fields are rather different. The core of the [100] screw dislocation is spread in an ellipsoid with the long axis along the (011) plane containing tetrahedrally coordinated magnesium atoms. The dislocation is spread $\sim 12 \AA$ along this plane while its thickness perpendicular to it is much more limited. However, there is some evidence for limited spreading in (010). Deformation associated with 
the core of the [001] screw dislocation is more isotropic with an elliptical cross section extending approximately $10 \AA$ in the [100] direction and $\sim 7 \AA$ in the [010] direction.

On the longest length-scale it is useful to consider the movements of the atoms during the geometry optimization. These movements are due to the model crystal not being a linear elastic continuum and can be represented as displacements parallel and perpendicular to the dislocation line (Figures 7 and 8). The displacement parallel to the dislocation line is smaller for the [100] than the [001] screw dislocation in both absolute terms and in terms of a fraction of the Burgers vector. In both cases the displacement rapidly decays away from the dislocation line and the magnesium ions move most. For both dislocations, the radial component of the displacement field is considerably smaller and decays more rapidly than the displacement parallel to the dislocation line. Movement inwards, towards the dislocation dominates. Only a very small number of atoms close to the core move outwards.

Figure 9 shows the energy of the two dislocation structures as a function of radius evaluated for radii between 2 and $49 \AA$ and plotted as circles. It is clear that the formation energy of the [001] screw dislocation is higher than the energy of the [100] dislocation. Two approaches are used to extract the core energy. The first approach involves fitting the energies to Equation 3 to give the lines in Figure 9 and the energy of the dislocation core. Assuming a core radius of $10 \AA$ (because the core energy and radius cannot be simultaneously determined, and this value allows a comparison 
with previous work) results in pre-logarithmic core energies of 2.48 and 4.45 $\mathrm{eV} / \AA$ for the [100] and [001] dislocations, respectively. In fitting the dislocation energy $K$ was taken as a free parameter, but for both dislocations the fitted value was within $1 \mathrm{GPa}$ of that expected from anisotropic elasticity and the calculated elastic constants. (For the [100] dislocation the $K$ was fitted as 106.2 GPa while elastic theory predicts 106.4 GPa. For [001] the fitted value was 100.7 GPa and the value from anisotropic elasticity was 101.4 GPa.) The second approach involves plotting the difference between the total energy and the elastic part of the energy (using $K$ from the elastic constants) this is shown in Figure 10 and converges to the core energy at large radius (Clouet, 2009). Using this approach gives somewhat smaller core energies and implies smaller core radii $(\sim 1.4 \mathrm{eV} / \AA$ and $\sim 6 \AA$ for the [100] dislocation, and $\sim 3.4 \mathrm{eV} / \AA$ and and $\sim 8 \AA$ for the [001] dislocation).

\section{Discussion and Conclusions}

The similarity of $K$ for the two dislocations emphasizes the rather low elastic anisotropy of wadsleyite and shows that the difference in (elastic) energy between the two dislocations is entirely due to the difference in length of the Burgers vectors. It is instructive to compare the core energy of the [100] screw dislocation with that of the $\langle 100\rangle$ screw dislocation in $\mathrm{MgO}$, a structurally simple dislocation core with an anomalously low core energy of $\sim 4.2 \mathrm{eV} / \AA$ (Watson et al., 1996, 1999; Walker et al., 2005b). Although the dislocation in $\mathrm{MgO}$ has a $25 \%$ shorter Burgers vector than the [100] 
screw dislocation in wadsleyite its core energy is $\sim 1.7$ times larger, a feature not explained by the higher shear modulus of $\mathrm{MgO}$. This supports the suggestion of a particularly stable core of the [100] screw dislocation made by Thurel et al. (2003b). A possible explanation for the low energy core is the structural flexibility offered by the presence of an oxygen atom that is not bonded to $\mathrm{Si}$ in the wadsleyite structure. Movement of this atoms and the surrounding $\mathrm{Mg}$ atoms parallel to the dislocation line can permit a smoothing out of the discontinuity in the elastic displacement field and allow the $\mathrm{Si}_{2} \mathrm{O}_{7}$ groups to relax to an unstrained low energy configuration.

The radial displacements shown in Figures 7(a) and 8(a) merit further discussion. The atoms move inwards, towards the dislocation core and this motion is completely absent in the linear elastic displacement field where the only non-zero components are parallel to the dislocation line (Equation 1; but note that for sufficiently low symmetry cases cross terms in the compliance matrix do introduce radial displacements). Similar patterns of inwards displacements have also been observed for screw dislocations in $\mathrm{MgO}$, forsterite (Walker et al., 2005b) and zeolite A (Walker et al., 2004) and it appears that the explanation may be rather general. Consider any circle threaded by the incipient dislocation line in the bulk material. This circle becomes a helix with the same radius once the elastic displacements have been applied and the dislocation introduced. Atomic separations along the circumference of the helix are increased compared to the separation in the bulk crystal structure (the circumference of a helix is longer then that 
of a circle with the same radius). One way of reducing the strain energy associated with this distortion, and moving towards the equilibrium atomic separation in the dislocated crystal, is to reduce the radius of the helix when compared with that of the original circle. This reduction can be accomplished by an inwards motion of the atoms surrounding the dislocation, as observed for both screw dislocations. Essentially, by reducing the volume of the dislocation core, the strain outside the core is reduced. For the [100] screw dislocation this reduction in core volume may also explain the occurrence of magnesium in tetrahedral coordination in the dislocation core. By moving from octahedral to tetrahedral coordination the volume occupied by the magnesium ions is reduced, this will reduce the volume of the dislocation core and allow atoms outside the core to move inwards to further reduce the elastic strain energy associated with this dislocation.

The atomic scale view of the core structure of the two dislocations provides an explanation for their different occurrences following deformation experiments. The fact that [100] dislocations are commonly found suggests that they are highly mobile allowing these dislocations to rapidly multiply during deformation. Conversely, the rarity of [001] dislocations suggests a much lower mobility. The structures point to a simple explanation for the different mobilities as movement on the [001] screw dislocation in any direction will inevitably involve the breaking and reforming $\mathrm{Si}-\mathrm{O}$ bonds. This processes will be associated with a large energy penalty preventing easy movement. On the other hand, movement of the [100] screw disloca- 
tion will not involve this high energy process. The required changes in the coordination of magnesium ions are likely to be associated with a lower energy barrier. The spreading of the [100] screw dislocation along the $\{011\}$ is suggestive of a particularly high mobility on this plane.

\section{Acknowledgments}

This work made use of the facilities of HECToR, the UK's national highperformance computing service, which is provided by UoE HPCx Ltd at the University of Edinburgh, Cray Inc and NAG Ltd, and funded by the Office of Science and Technology through EPSRC's High End Computing Programme. I gratefully acknowledge the support of the NERC through a Postdoctoral Research Fellowship (NE/E012922/2) and thank two anonymous reviewers for useful comments.

\section{References}

Ahrens TJ (1995) Mineral Physics and Crystallography, a Handbook of Physical Constants. Vol. 2 of AGU Reference Shelf. AGU.

Carrez P, Cordier D, Mainprice D, Tommasi A (2006) Slip systems and plastic shear anisotropy in $\mathrm{Mg}_{2} \mathrm{SiO}_{4}$ ringwoodite: insights from numerical modelling. European Journal of Mineralogy 18:149-160.

Carrez P, Ferré D, Cordier D (2007a) Implications for plastic flow in the deep mantle from modelling dislocations in $\mathrm{MgSiO}_{3}$ minerals. Nature 446:6870. 
Carrez P, Ferré D, Cordier D (2007b) Peierls-Nabarro model for dislocations in $\mathrm{MgSiO}_{3}$ post-perovskite calculated at $120 \mathrm{GPa}$ from first principles. Philosophical Magazine 87:3229-3247.

Carrez P, Walker AM, Metsue A, Cordier D (2008) Evidence from numerical modelling for 3D spreading of [001] screw dislocations in $\mathrm{Mg}_{2} \mathrm{SiO}_{4}$ forsterite. Philosophical Magazine 88:2477-2485.

Chen J, Inoue T, Weidner DJ, Wu Y, Vaughan MT (1998). Strength and water weakening of mantle minerals, olivine, wadsleyite and ringwoodite. Geophysical Research Letters 25:575-578.

Clouet E, (2009) Elastic energy of a straight dislocation and contribution from core tractions. Philosophical Magazine 89:1565-1584.

Clouet E, Ventelon L, Willaime F (2009) Dislocation core energies and core fields from first principles. Physical Review Letters 102, art.num.055502. de Leeuw NH, Parker SC, Catlow CRA, Price GD (2000) Proton-containing defects at forsterite $\{010\}$ tilt grain boundaries and stepped surfaces. American Mineralogist 85:1143-1154.

Dupas C, Doukhan N, Doukhan JC, Green HW, Young TE (1994) Analytical electron microscopy of a synthetic peridotite experimentally deformed in the $\beta$ olivine stability field. Journal of Geophysical Research 99:1582115832.

Dupas-Bruzek C, Sharp TG, Rubie DC, Durham WB (1998) Mechanisms of transformation and deformation in $\mathrm{Mg}_{1.8} \mathrm{Fe}_{0.2} \mathrm{SiO}_{4}$ olivine and wadsleyite under non-hydrostaric stress. Physics of the Earth and Planetary Interiors 
108:33-48.

Durinck J, Carrez P, Cordier P (2007) Application of the peierls-nabarro model to dislocations in forsterite. European Journal of Mineralogy 19:631-639.

Durinck J, Legris A, Cordier P (2005a) Influence of crystal chemistry on ideal plastic shear anisotropy in forsterite: First principle calculations. American Mineralogist 90:1072-1077.

Durinck J, Legris A, Cordier P (2005b) Pressure sensitivity of olivine slip systems: first-principle calculations of generalised stacking faults. Physics and Chemistry of Minerals 32:646-654.

Fennell CJ, Gezelter JD (2006) Is the Ewald summation still necessary? Journal of Chemical Physics, 124, art.no.23404.

Ferré D, Carrez P, Cordier P (2007) First principles determination of dislocations properties of $\mathrm{MgSiO}_{3}$ perovskite at $30 \mathrm{GPa}$ based on the PeierlsNabarro model. Physics of the Earth and Planetary Interiors 163:283-291.

Ferré D, Carrez P, Cordier P (2008) Modelling dislocation cores in $\mathrm{SiTiO}_{3}$ using the Peierls-Nabaro model. Physical Review B, 77, art.no.014106.

Ferré D, Carrez P, Cordier P (2009a) Dislocation modeling in calcium silicate perovskite based on the Peierls-Nabarro model. American Mineralogist 94:135-142.

Ferré D, Carrez P, Cordier P (2009b) Peierls dislocation modelling in perovskite $\left(\mathrm{SaTiO}_{3}\right)$ : comparison with tausonite $\left(\mathrm{SrTiO}_{3}\right)$ and $\mathrm{MgSiO}_{3}$ perovskite. Physics and Chemistry of Minerals 36:233-239. 
Gale JD (1996) Empirical potential derivation for ionic materials. Philosophical Magazine B 73:3-19.

Gale JD (1997) GULP: A computer program for the symmetry-adapted simulation of solids. Journal of the Chemical Society, Faraday Transactions 93:629-637.

Gale JD, Rohl AL (2003) The general utility lattice program (GULP). Molecular Simulation 29:291-341.

Jackson, I., 2007. Properties of rocks and minerals. In: Price, G. D. (Ed.), Physical Origins of Anelasticity and Attenuation in Rocks. Vol. 2 of Treatise on Geophysics. Elsevier, pp. 493-525.

Jia CL, Thust A, Urban K (2005) Atomic-scale analysis of the oxygen configuration at a $\mathrm{SrTiO}_{3}$ dislocation core. Physical Review Letters 95, art.num.225506.

Johnson CL, Hÿtch MJ, Buseck PR (2004) Displacement and strain fields around a [100] dislocation in olivine measured to sub-angstrom accuracy. American Mineralogist 89:1374-1379.

Karato S-I, Spetzler HA (1990) Defect microdynamics in minerals and solid state mechanisms of seismic wave attenuation and velocity dispersion in the mantle. Reviews of Geophysics 28:399-421.

Kerschhofer L, Dupas C, Liu M, Sharp TG, Durham WB, Rubie DC (1998) Polymorphic transformations between olivine to wadsleyite and ringwoodite: mechanisms of intracrystalline nucleation and the role of elastic strain. Mineralogical Magazine 62:617-638. 
Kerschhofer L, Sharp TG, Rubie DC (1996) Intracrystalline transformation of olivine to wadsleyite and ringwoodite under subduction zone conditions. Science 274:79-81.

Kumazawa M, Anderson OL (1969) Elastic moduli, pressure derivatives, and temperature derivatives of single-crystal olivine and single-crystal forsterite. Journal of Geophysical Research 74:5961-5972.

Lewis GV, Catlow CRA (1985) Potential models for ionic oxides. Journal of Physics C: Solid State Physics 18:1149-1161.

Madon M, Poirier JP (1983) Transmission electron microscope observation of $\alpha, \beta$ and $\gamma(\mathrm{Mg}, \mathrm{Fe})_{2} \mathrm{SiO}_{4}$ in shocked meteorites planar defects and polymorphic transitions. Physics of the Earth and Planetary Interiors $33: 31-44$.

Montagner JP, Kennett BLN (1996) How to reconcile body-wave and normal-mode reference earth models. Geophysics Journal International 125:229-248.

Mossenfelder JL, Connolly JAD, Rubie DC, Liu M (2000) Strength of $(\mathrm{Mg}, \mathrm{Fe})_{2} \mathrm{SiO}_{4}$ wadsleyite determined by relaxation of transformation stress. Physics of the Earth and Planetary Interiors 120:63-78.

Nabarro FRN (1947) Dislocations in a simple cubic lattice. Proceedings of the Physical Society of London 59:256-272.

Nishihara Y, Tinker D, Kawazoe T, Xu Y, Jing Z, Matsukage KN, Karato S-I (2008) Plastic deformation of wadsleyite and olivine at high-pressure and high-temperature using a rotational Drickamer apparatus (RDA). 
Physics of the Earth and Planetary Interiors 170:156-169.

Nocedal J (1980) Updating quasi-Newton matrices with limited storage. Mathematics of Computation 25:773-782.

Peierls RE (1940) On the size of a dislocation. Proceedings of the Physical Society of London 52:34-37.

Price GD (1983) The nature and significance of stacking faults in wadsleyite, natural $\beta-(\mathrm{Mg}, \mathrm{Fe})_{2} \mathrm{SiO}_{4}$ from the Peace River meteorite. Physics of the Earth and Planetary Interiors 33:137-147.

Price GD, Putnis A, Smith D (1982) A spinel to $\beta$-phase transformation mechanism in $(\mathrm{Mg}, \mathrm{Fe})_{2} \mathrm{SiO}_{4}$. Nature 296:729-731.

Purton JA, Allan NL, Blundy JD (1997) Calculated energies of heterovalent cations in forsterite and diopside: implications for trace element partitioning. Geochimica et Cosmochimica Acta 61:3927-3936.

Sanders MJ, Leslie M, Catlow CRA (1984) Interatomic potentials for $\mathrm{SiO}_{2}$. Journal of the Chemical Society, Chemical Communications, 1271 - 1273. doi:10.1039/C39840001271

Sawamoto H, Weidner DJ, Sasaki S, Kumazawa M (1984) Single-crystal elastic properties of the modified spinel (beta) phase of magnesium orthosilicate. Science 224:749-751.

Schoeck G (2005) The peierls model: Progress and limitations. Materials Science and Engineering A. 400-401:7-17.

Sharp TG, Bussod GYA, Katsura T (1994) Microstructures in beta$\mathrm{Mg}_{1.8} \mathrm{Fe}_{0.2} \mathrm{SiO}_{4}$ experimentally deformed at transition-zone conditions. 
Physics of the Earth and Planetary Interiors 86:69-83.

Steeds JW, (1973) Introduction to anisotropic theory of dislocations. Oxford University Press, Oxford.

Thurel E, Cordier P (2003) Plastic deformation of wadsleyite: I. high pressure deformation in compression. Physics and Chemistry of Minerals $30: 256-266$.

Thurel E, Cordier P, Frost D, Karato S-I (2003a) Plastic deformation of wadsleyite: II. high pressure deformation in shear. Physics and Chemistry of Minerals 30:267-270.

Thurel E, Douin J, Cordier P (2003b) Plastic deformation of wadsleyite: III. interpretation of dislocations and slip systems. Physics and Chemistry of Minerals 30:271-279.

Tommasi A, Mainprice D, Cordier P, Thoraval C, Couvy H (2004) Straininduced seismic anisotropy of wadsleyite polycrystals and flow patterns in the mantle transition zone. Journal of Geophysical Research 109, art.no.B12405.

Trampert J, van Heijst HJ (2002) Global azimuthal anisotropy in the transition zone. Science 29:1297-1299.

Vitek V, Perrin R, Bowen D (1970) The core structure of $1 / 2\langle 111\rangle$ screw dislocations in b.c.c. crystals. Philosophical Magazine A 21:1049-1073.

Walker AM, Demouchy S, Wright K (2006) Computer modelling of the energies and vibrational properties of hydroxyl groups in alpha- and beta$\mathrm{Mg}_{2} \mathrm{SiO}_{4}$. European Journal of Mineralogy 18:529-543. 
Walker AM, Gale JD, Slater B, Wright K (2005a) Atomic scale modelling of the cores of dislocations in complex materials part 1: methodology. Physical Chemistry Chemical Physics 7:3227-3234.

Walker AM, Gale JD, Slater B, Wright K (2005b) Atomic scale modelling of the cores of dislocations in complex materials part 2: applications. Physical Chemistry Chemical Physics 7:3235-3242.

Walker AM, Slater B, Gale JD, Wright K (2004) Predicting the structure of screw dislocations in nanoporous materials. Nature Materials 3:715-720.

Walker AM, Woodley SM, Slater B, Wright K (2009) A computational study of magnesium point defects and diffusion in forsterite. Physics of the Earth and Planetary Interiors 172:20-27.

Walker AM, Wright K, Slater B (2003) A computational study of oxygen diffusion in olivine. Physics and Chemistry of Minerals 30:536-545.

Watson GW, Kelsey ET, de Leeuw NH, Harris DJ, Parker SC (1996) Atomistic simulation of dislocations, surfaces and interfaces in MgO. Journal of the Chemical Society, Faraday Transactions 92:433-438.

Watson GW, Kelsey ET, Parker SC (1999) Atomistic simulation of screw dislocations in rock salt structured materials. Philosophical Magazine A 79:527-536.

Wolf D, Keblinski P, Phillpot SR, Eggebrecht J (1999) Exact method for the simulation of Coulombic systems by spherically truncated, pairwise $r^{-1}$ summation. Journal of Chemical Physics 110:8254-8282. 
Zha C, Mao H, Hemley RJ (1997) Elasticity of MgO and a primary pressure scale to $55 \mathrm{GPa}$. Proceedings of the National Acadamy of Science 97:13494-13499. 


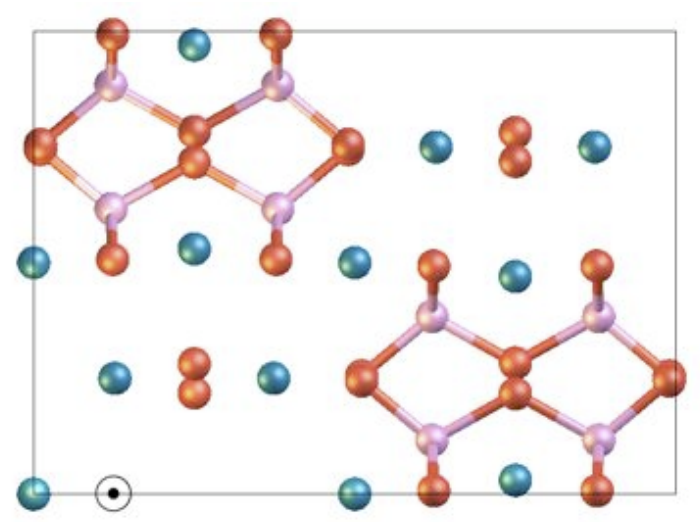

Fig. 1 One unit cell of the bulk structure of wadsleyite, looking along the [100] direction. The [010] direction is horizontal and the [001] direction is vertical. The location of the incipient [100] screw dislocation line is marked with two concentric circles. Si atoms are shown in pink, $\mathrm{O}$ atoms are shown in red and $\mathrm{Mg}$ atoms are shown in blue.

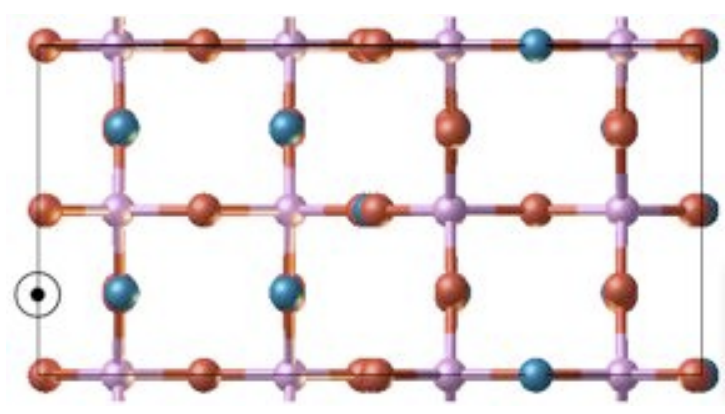

Fig. 2 One unit cell of the bulk structure of wadsleyite, looking along the [001] direction. The [010] direction is horizontal and the [100] direction is vertical. The location of the incipient [001] screw dislocation line is marked with two concentric circles. Si atoms are shown in pink, $\mathrm{O}$ atoms are shown in red and $\mathrm{Mg}$ atoms are shown in blue. 


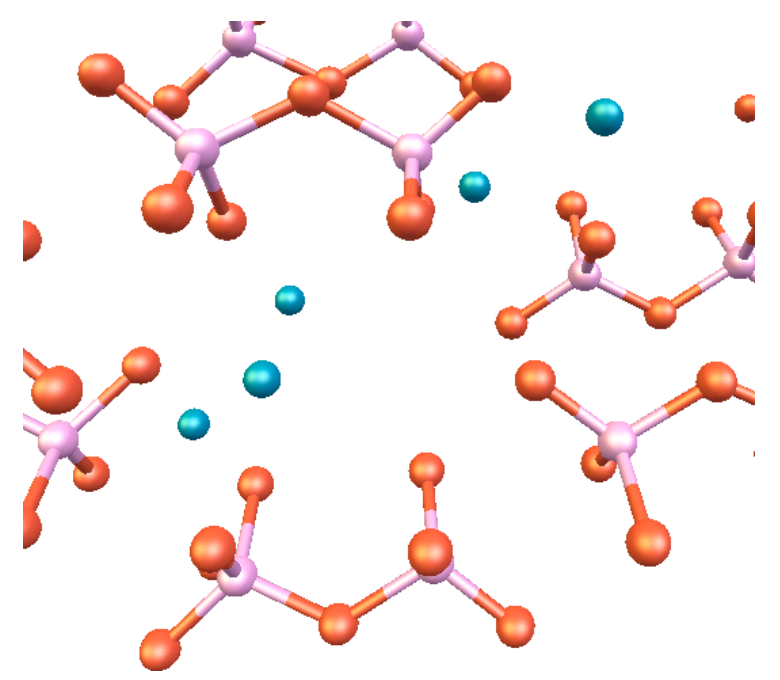

Fig. 3 Perspective atomic scale views of the core of the [100] dislocation looking along the dislocation line. Only $\mathrm{SiO}_{4}$ tetrahedra (pink: $\mathrm{Si}$, red: O) and 4coordinated $\mathrm{Mg}$ (blue) are shown.

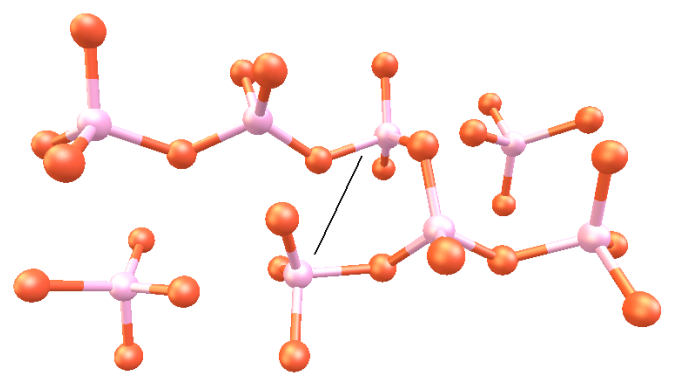

Fig. 4 Perspective atomic scale views of the cores of the [001] dislocation viewed oblique to the dislocation line, marked with the black line. Only $\mathrm{SiO}_{4}$ tetrahedra (pink: Si, red: O) are shown. 


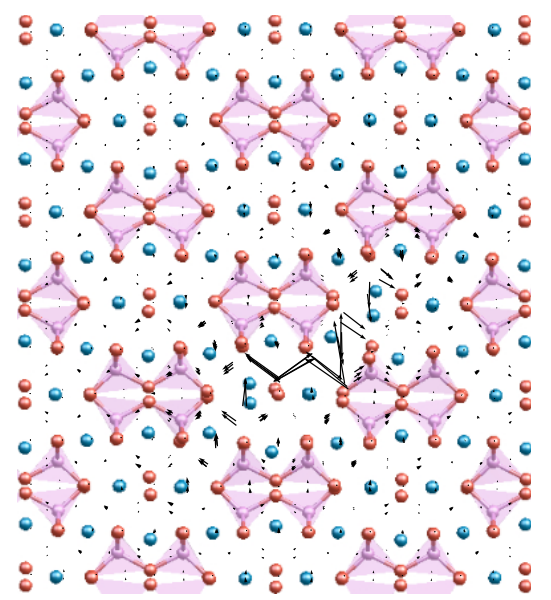

Fig. 5 Differential displacement plots of the [100] screw dislocation. Arrows show difference in displacement of adjacent oxygen atoms along the dislocation line (see text) superimposed on the structure around dislocation core. Width $23 \AA$. 


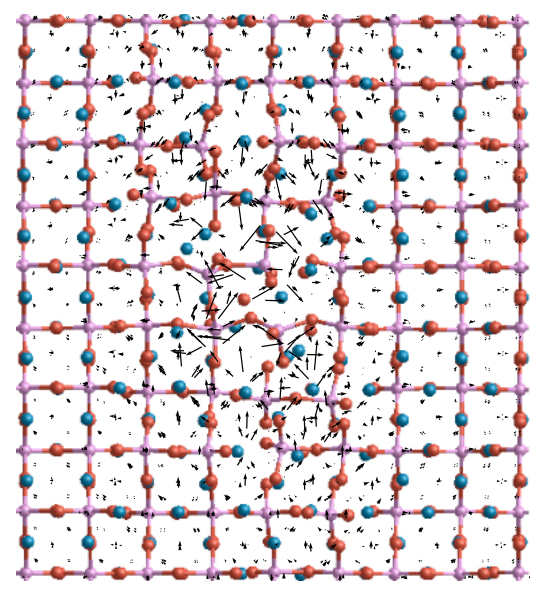

Fig. 6 Differential displacement plots of the [001] screw dislocation. Arrows show difference in displacement of adjacent oxygen atoms along the dislocation line (see text) superimposed on the structure around dislocation core. Width $23 \AA$. 


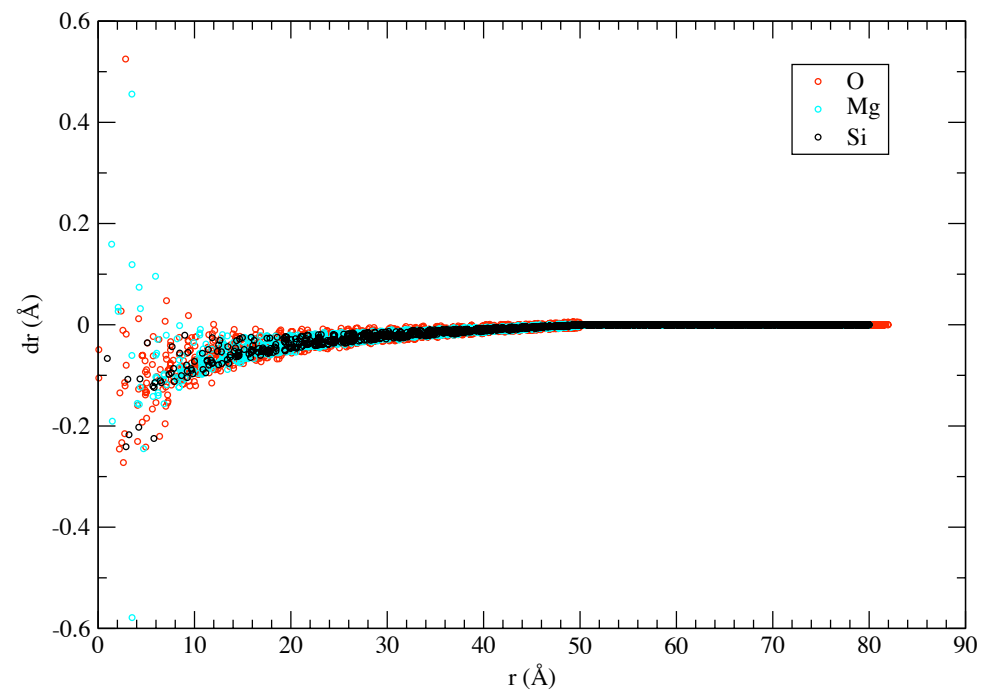

(a)

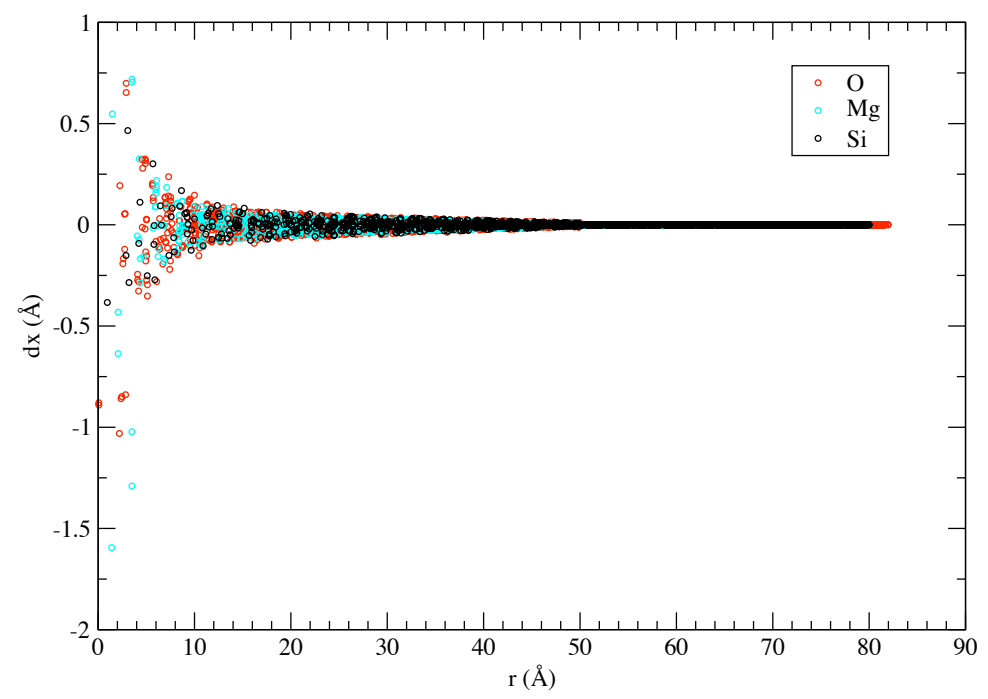

(b)

Fig. 7 Non-elastic displacement field of [100] screw dislocations plotted for each atom in the simulation cell. Part (a) shows the radial displacements (with positive displacements being away from the dislocation line) and part (b) shows displacements parallel to the dislocation line. 


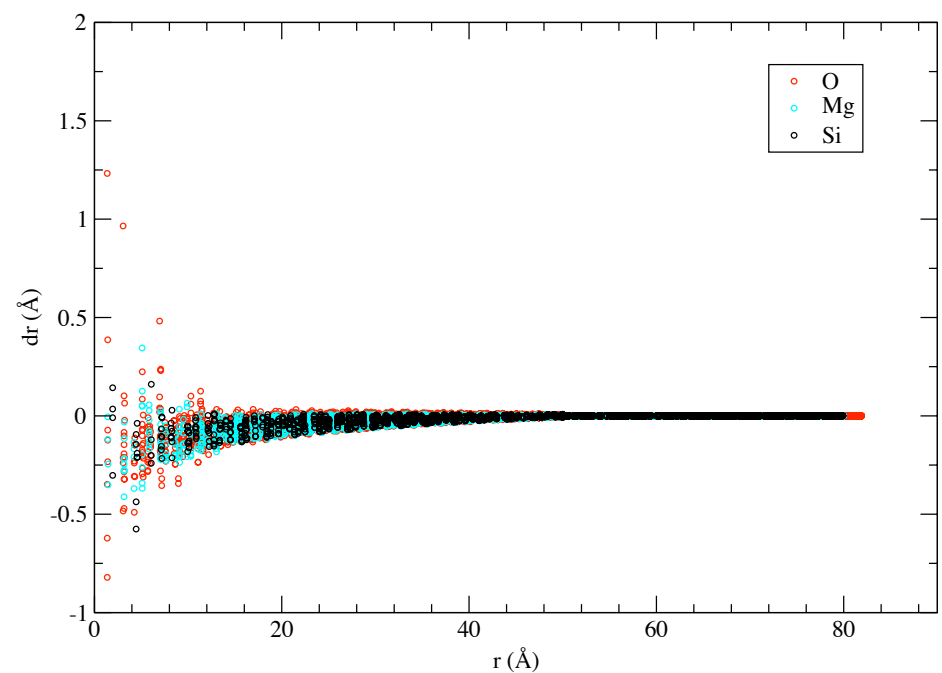

(a)

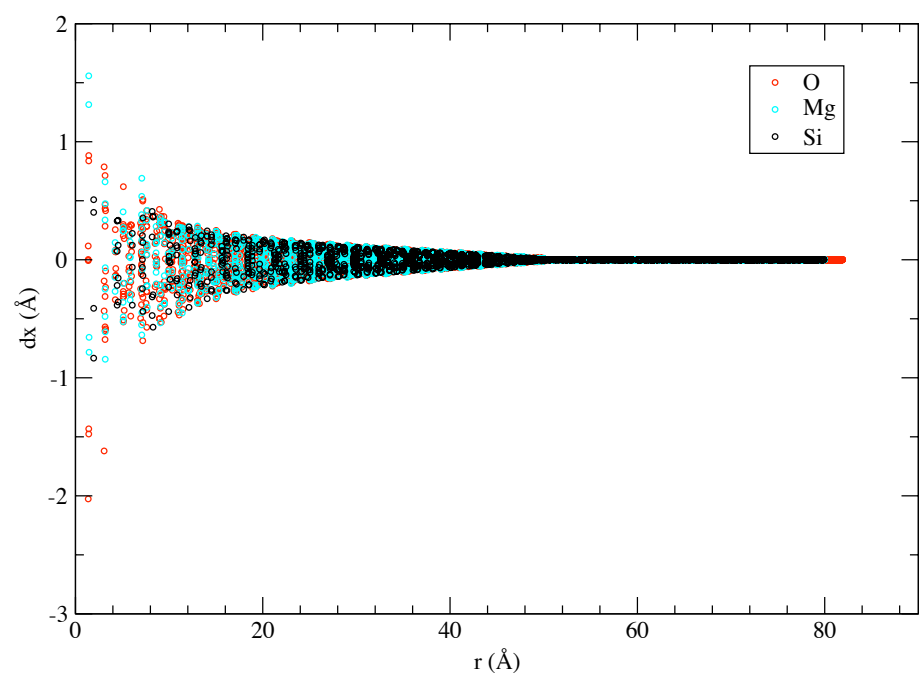

(b)

Fig. 8 Non-elastic displacement field of [001] screw dislocations plotted for each atom in the simulation cell. Part (a) shows the radial displacements (with positive displacements being away from the dislocation line) and part (b) shows displacements parallel to the dislocation line. 


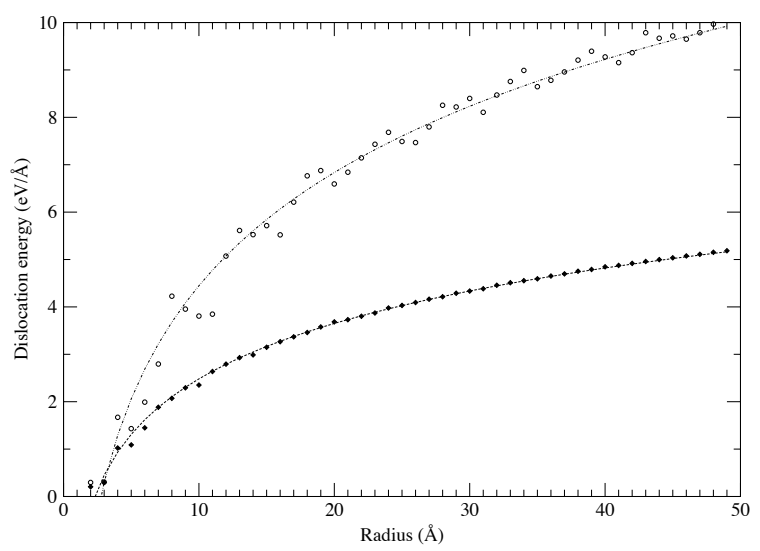

Fig. 9 Dislocation formation energy as a function of radius from dislocation line for [100] (solid symbols) and [001] (open symbols). Lines are fits to Equation 3.

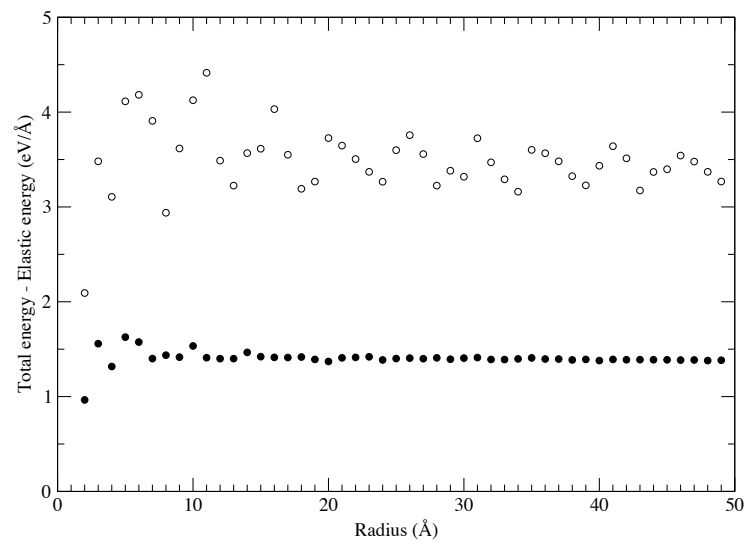

Fig. 10 Difference between the total and elastic part of the dislocation formation energy as a function of radius from dislocation line for [100] (solid symbols) and [001] (open symbols). 
Table 1 Parameters of the fitted interatomic potential model. Buckingham potentials operate between atoms located less than $8.0 \AA$ apart, the three body potential operates between $\mathrm{O}-\mathrm{Si}-\mathrm{O}$ groups where the $\mathrm{O}-\mathrm{O}$ distance is less than $8.0 \AA$ and the $\mathrm{Si}-\mathrm{O}$ distance is less than $4.0 \AA$. Short range interactions are tapered between 7.0 and $8.0 \AA$ A. Coulomb interactions, treated using the Wolf summation (Wolf et al., 1999), are truncated at a radius of $16.0 \AA$ and damped using an $\eta$ parameter of $0.2 \AA^{-1}$.

\begin{tabular}{llll}
\hline Buckingham & $A(\mathrm{eV})$ & $\rho(\AA)$ & $C\left(\mathrm{eV} . \AA^{6}\right)$ \\
$\mathrm{Si}-\mathrm{O}$ & 1443.6999 & 0.308 & 10.66 \\
$\mathrm{Mg}-\mathrm{O}$ & 1396.9531 & 0.149 & 0.0 \\
$\mathrm{O}-\mathrm{O}$ & 22764.000 & 0.149 & 27.88 \\
\hline Three body exponential & $k\left(\mathrm{eV}^{\mathrm{r}} \mathrm{rad}^{-2}\right)$ & $\theta_{0}\left(^{\circ}\right)$ & $\rho_{\text {SiO }}(\AA)$ \\
$\mathrm{O}-\mathrm{Si}-\mathrm{O}$ & $0.671854 \times 10^{8}$ & 109.47 & 0.180 \\
\hline Polarizability & Core charge & Shell charge & $k_{c s}\left(\mathrm{eV} . \AA^{-2}\right)$ \\
O & 0.8482 & -2.8482 & 74.92 \\
\hline
\end{tabular}


Table 2 Calculated and experimental lattice parameters and properties of $\mathrm{MgO}$, forsterite and wadsleyite. Experimental data from Sawamoto et al. (1984), Ahrens (1995), Kumazawa and Anderson (1969), and Zha et al. (1997). "Previous model" refers to model of Sanders et al. (1984) and Lewis and Catlow (1985)

\begin{tabular}{|c|c|c|c|}
\hline $\mathrm{MgO}$ & Experiment & Previous model & This work \\
\hline $\mathrm{a}$ & 4.212 & 4.199 & 4.233 \\
\hline$c_{11}$ & $297.0 \pm 0.1$ & 392.82 & 376.85 \\
\hline $\mathrm{c}_{12}$ & $95.2 \pm 0.7$ & 164.21 & 159.46 \\
\hline $\mathrm{c}_{44}$ & $155.7 \pm 0.7$ & 164.21 & 159.46 \\
\hline$\epsilon^{0}$ & 9.86 & 8.38 & 8.47 \\
\hline$\epsilon^{\infty}$ & 2.96 & 2.17 & 2.14 \\
\hline \multicolumn{4}{|c|}{$\alpha-\mathrm{Mg}_{2} \mathrm{SiO}_{4}$} \\
\hline $\mathrm{a}$ & 4.75 & 4.7818 & 4.7596 \\
\hline $\mathrm{b}$ & 10.19 & 10.2464 & 10.3385 \\
\hline $\mathrm{c}$ & 5.98 & 5.9863 & 6.0313 \\
\hline $\mathrm{c}_{11}$ & 328 & 358.62 & 330.09 \\
\hline $\mathrm{c}_{22}$ & 200 & 206.62 & 205.83 \\
\hline $\mathrm{c}_{33}$ & 235 & 281.14 & 278.14 \\
\hline $\mathrm{c}_{12}$ & 64 & 93.83 & 94.67 \\
\hline $\mathrm{c}_{13}$ & 69 & 96.18 & 99.44 \\
\hline $\mathrm{c}_{23}$ & 74 & 87.70 & 90.20 \\
\hline $\mathrm{c}_{44}$ & 66 & 44.23 & 45.92 \\
\hline $\mathrm{c}_{55}$ & 81 & 74.54 & 76.69 \\
\hline $\mathrm{c}_{66}$ & 81 & 84.29 & 83.42 \\
\hline \multicolumn{4}{|c|}{$\beta-\mathrm{Mg}_{2} \mathrm{SiO}_{4}$} \\
\hline $\mathrm{a}$ & 5.69 & 5.6515 & 5.6561 \\
\hline $\mathrm{b}$ & 11.46 & 11.3908 & 11.3384 \\
\hline c & 8.25 & 8.2746 & 8.2999 \\
\hline $\mathrm{c}_{11}$ & $360 \pm 6$ & 434.60 & 433.05 \\
\hline $\mathrm{c}_{22}$ & $383 \pm 4$ & 425.65 & 415.27 \\
\hline$c_{33}$ & $273 \pm 5$ & 331.68 & 332.34 \\
\hline $\mathrm{c}_{12}$ & $75 \pm 9$ & 118.61 & 124.28 \\
\hline $\mathrm{c}_{13}$ & $110 \pm 6$ & 136.18 & 133.52 \\
\hline $\mathrm{c}_{23}$ & $105 \pm 9$ & 144.10 & 141.08 \\
\hline $\mathrm{c}_{44}$ & $112 \pm 2$ & 101.61 & 92.91 \\
\hline$c_{55}$ & $118 \pm 4$ & 113.47 & 110.67 \\
\hline $\mathrm{c}_{66}$ & $98 \pm 4$ & 100.57 & 102.26 \\
\hline
\end{tabular}

